\title{
Numerical solution of Riemann-Hilbert problems: Painlevé II
}

\author{
Sheehan Olver
}

\begin{abstract}
We describe a new spectral method for solving matrix-valued Riemann-Hilbert problems numerically. We demonstrate the effectiveness of this approach by computing solutions to the homogeneous Painlevé II equation. This can be used to relate initial conditions with asymptotic behaviour.
\end{abstract}

Keywords Riemann-Hilbert problems, spectral methods, Painlevé transcendents.

Oxford University Mathematical Institute

Numerical Analysis Group

24-29 St Giles'

Oxford, England OX1 3LB

E-mail: Sheehan.Olver@sjc.ox.ac.uk

December, 2009 


\section{Introduction}

Riemann-Hilbert problems occupy an important place in applied analysis. They have been used to derive the asymptotics of nonlinear differential equations such as the Painlevé transcendents [10], the nonlinear Schrödinger equation and the $\mathrm{KdV}$ equation [1] , as well as orthogonal polynomials and random matrices [7]. The key to their effectiveness is that they can be deformed in the complex plane to turn oscillations into exponential decay. This is known as nonlinear steepest descent [9], as it is much like the classical theory of steepest descent for oscillatory integrals. Thus Riemann-Hilbert formulations can loosely be viewed as a nonlinear counterpart to the integral representations that are known for many important linear differential equations; such as the Airy equation, hypergeometric equations [2], wave equation and heat equation.

Integral representations have another important use, in addition to the derivation of asymptotics: numerical computation through quadrature. Indeed, they have been used to great effect for computing Airy functions and Bessel functions [11], which, in a certain sense, are linear analogues of Painlevé transcendents. The aim of this paper is to demonstrate that Riemann-Hilbert problems share this property with integral representations: they can also be used to compute solutions to the associated equations numerically.

A Riemann-Hilbert problem is the problem of finding a function that is analytic everywhere in the complex plane except along a given curve, on which it has a prescribed jump. This can be written more precisely as

Problem 1.1 [14] Given an oriented curve $\Gamma \subset \mathbb{C}$ and a jump matrix $G: \Gamma \rightarrow \mathbb{C}^{2 \times 2}$, find a function $\Phi: \mathbb{C} \backslash \Gamma \rightarrow \mathbb{C}^{2 \times 2}$ which is analytic everywhere except on $\Gamma$ such that

$$
\begin{aligned}
& \Phi^{+}(z)=\Phi^{-}(z) G(z) \quad \text { for } z \in \Gamma \text { and } \\
& \Phi(\infty)=I
\end{aligned}
$$

where $\Phi^{+}$denotes the limit of $\Phi$ as $z$ approaches $\Gamma$ from the left, and $\Phi^{-}$denotes the limit of $\Phi$ as $z$ approaches $\Gamma$ from the right and $\Phi(\infty)=\lim _{|z| \rightarrow \infty} \Phi(z)$.

To demonstrate the numerical approach, we will focus on the Painlevé II transcendent, though the techniques developed are generalizable for other Riemann-Hilbert problems. The Painlevé II equation is

$$
u^{\prime \prime}=x u+2 u^{3}-\alpha,
$$

where $\alpha$ is a complex parameter. For simplicity, we will take $\alpha=0$. From this differential equation, an equivalent Riemann-Hilbert formulation is derived by finding a Lax pair representation, which in turn is uniquely specified by behaviour along Stokes' lines. This can then be rephrased as a Riemann-Hilbert problem [10]. 


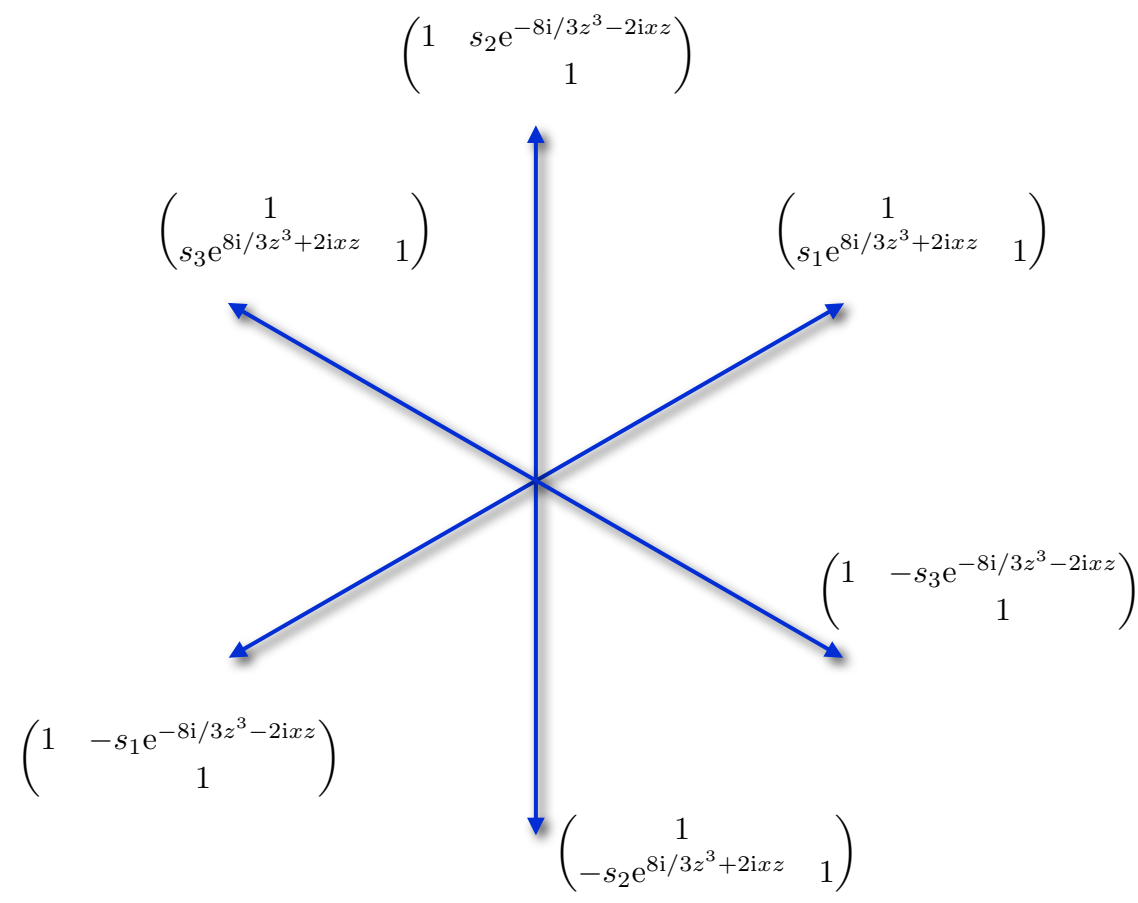

Figure 1: The curve and jump matrix for the Painlevé II Riemann-Hilbert problem.

As explained in $[\mathbf{1 2}]$, the curve $\Gamma$ in the Riemann-Hilbert problem for the homogeneous Painlevé II equation consists of six rays originating from the origin (see Figure 1):

$$
\begin{aligned}
\Gamma & =\Gamma_{1} \cup \cdots \cup \Gamma_{6} \\
\text { where } \quad \Gamma_{\kappa} & =\left\{z \in \mathbb{C}: \arg z=\frac{\pi}{6}+\frac{\pi}{3}(\kappa-1)\right\} .
\end{aligned}
$$

The jump function has a different definition depending on which ray $\Gamma_{\kappa}$ that $z$ lies on:

$$
G(x ; z)=\left\{\begin{array}{cc}
\left(\begin{array}{cc}
1 & s_{\kappa} \mathrm{e}^{-8 \mathrm{i} / 3 z^{3}-2 \mathrm{i} x z} \\
1
\end{array}\right) & \kappa \text { even and } z \in \Gamma_{\kappa}, \\
1 & \\
\left(\begin{array}{cc}
1 \\
s_{\kappa} \mathrm{e}^{8 \mathrm{i} / 3 z^{3}+2 \mathrm{i} x z} & 1
\end{array}\right) & \kappa \text { odd and } z \in \Gamma_{\kappa} .
\end{array}\right.
$$

Instead of imposing initial conditions, we choose constants $s_{1}, s_{2}$ and $s_{3}$ which satisfy the following compatibility condition:

$$
\begin{gathered}
s_{1}-s_{2}+s_{3}+s_{1} s_{2} s_{3}=0, \\
s_{4}=-s_{1}, s_{5}=-s_{2}, s_{6}=-s_{3} .
\end{gathered}
$$

Once we have found a function $\Phi(x ; z)$ that satisfies the Painlevé II Riemann-Hilbert problem, a solution to (1.1) is

$$
u(x)=\lim _{z \rightarrow \infty} 2 z \Phi^{(12)}(x ; z) .
$$


If $x$ is not such that $u(x)$ has a pole, then (1.2) is sufficient to ensure a unique solution to the Riemann-Hilbert problem [10]. As in the integral representation of the Airy function, the original variable $x$ has been reduced to a parameter.

By moving from the original differential equation to Problem 1.1, we have transformed a nonlinear problem to a linear problem, ignoring the boundary condition at $\infty$. We can rephrase the problem so that it is completely linear: define

$$
U=\Phi-I
$$

thence,

$$
\mathcal{L} U=U^{+}-U^{-} G=G-I \quad \text { and } \quad U(\infty)=0 .
$$

Now the operator $\mathcal{L}$ is linear, from the space of functions analytic off $\Gamma$ and which decay at $\infty$ to the space of functions defined on $\Gamma$.

Consider for the moment the following simple and scalar Riemann-Hilbert problem:

Problem 1.2 Given an oriented curve $\Gamma \subset \mathbb{C}$ and a function $f: \Gamma \rightarrow \mathbb{C}$ which is Höldercontinuous on each smooth segment of $\Gamma$, find a function $\psi: \mathbb{C} \backslash \Gamma \rightarrow \mathbb{C}$ which is analytic everywhere except on $\Gamma$ such that

$$
\psi^{+}(z)-\psi^{-}(z)=f(z) \quad \text { for } \quad z \in \Gamma \quad \text { and } \quad \psi(\infty)=0 .
$$

Definition 1.3 We denote the linear operator that maps $f$ to such a $\psi$ as $\mathcal{M}_{\Gamma}$, and the linear operators that map $f$ to $\psi^{ \pm}$as $\mathcal{M}_{\Gamma}^{ \pm}$. When $\Gamma$ is clear from context, we use the notation $\mathcal{M}$.

For analysts, solving this equation is a trivial application of Plemelj's lemma [14], and can be written in terms of the Hilbert transform:

$$
\mathcal{M} f(z)=\frac{1}{\mathrm{i} \pi} \int_{\Gamma} \frac{f(t)}{z-t} \mathrm{~d} t \quad \text { for } \quad z \notin \Gamma .
$$

Though the Hilbert transform can be computed using many methods (cf. [13] for a recent review), in a certain sense we have moved backwards: the functions in Problem 1.2 are bounded (and, in the Painlevé case, analytic), whereas (1.4) has introduced a singularity that must somehow be dealt with. Thus the approach taken in [17] was to apply Plemelj's lemma in reverse; i.e., compute (1.4) numerically by rewriting it as Problem 1.2. This can be accomplished efficiently using the FFT when $\Gamma$ is a circle, interval, ray or combination of multiple such curves, as reviewed in Section 3. Moreover, the computation of $\mathcal{M}$ is achieved by computing $\mathcal{M}^{ \pm}$, which is what we actually require.

The importance of the operator $\mathcal{M}$ is that it uniquely maps any Hölder-continuous function defined on $\Gamma$ to an analytic function defined off $\Gamma$. This still holds true when it is a matrix-valued function, in a component-wise manner. Moreover, under certain conditions 
which are satisfied in our case, this map is one-to-one. In other words, for a function $V$ defined on $\Gamma$, if

$$
\mathcal{L} \mathcal{M} V=\mathcal{M}^{+} V-\left(\mathcal{M}^{-} V\right) G=G-I
$$

it follows that $\Psi=\mathcal{M} V+I$ satisfies all the conditions of Problem 1.1. Moreover, $\mathcal{L} \mathcal{M}$ maps the space of functions which are Hölder-continuous on the segments of $\Gamma$ to itself.

The numerical method can now be described as follows, and it is essentially a collocation method:

\section{Algorithm 1.4}

1: $\quad$ Represent

$$
V(z)=\left\{\begin{array}{ll}
V_{1}(z) & z \in \Gamma_{1} \\
& \vdots \\
V_{6}(z) & z \in \Gamma_{6}
\end{array} \quad \text { for } \quad V_{\kappa}(z)=\left(\begin{array}{ll}
\boldsymbol{p}_{\kappa}(z) \boldsymbol{v}_{\kappa}^{(11)} & \boldsymbol{p}_{\kappa}(z) \boldsymbol{v}_{\kappa}^{(12)} \\
\boldsymbol{p}_{\kappa}(z) \boldsymbol{v}_{\kappa}^{(21)} & \boldsymbol{p}_{\kappa}(z) \boldsymbol{v}_{\kappa}^{(22)}
\end{array}\right)\right.
$$

where $\boldsymbol{p}_{\kappa}=\left(p_{\kappa 1}, \ldots, p_{\kappa n}\right)$ denotes a basis for functions defined on $\Gamma_{\kappa}$ and $\boldsymbol{v}_{\kappa}^{(i j)}=$ $\left(v_{\kappa 1}^{(i j)}, \ldots, v_{\kappa n}^{(i j)}\right)^{\top}$ is in $\mathbb{C}^{n}$

2: $\quad$ For collocation points $\boldsymbol{z}_{\kappa}=\left(z_{\kappa 1}, \ldots, z_{\kappa n}\right)^{\top}$ in $\Gamma_{\kappa}$, compute $\mathcal{M}^{ \pm} V\left(\boldsymbol{z}_{\kappa}\right)$ for $\kappa=1, \ldots, 6$;

3: $\quad$ Determine $\boldsymbol{v}_{\kappa}^{(i j)}$ by solving the $24 n \times 24 n$ linear system

$$
\begin{aligned}
\mathcal{L M} V\left(\boldsymbol{z}_{1}\right) & =G\left(\boldsymbol{z}_{1}\right)-I \\
& \vdots \\
\mathcal{L} \mathcal{M} V\left(\boldsymbol{z}_{6}\right) & =G\left(\boldsymbol{z}_{6}\right)-I
\end{aligned}
$$

4: $\quad$ Convert $\left(\boldsymbol{v}_{1}^{(12)}, \ldots, \boldsymbol{v}_{6}^{(12)}\right)^{\top}$ to $u_{n}(x)$ which approximates $u(x)$.

Though this is set-up in a framework which is applicable to a general class of RiemannHilbert problems, we will focus on constructing the scheme for the homogeneous Painlevé II equation. The next four sections correspond to each of the steps of Algorithm 1.4. Whereas Algorithm 1.4 suggests that we will need to solve a $24 n \times 24 n$ linear system, we will find that the degree of the linear system will only be $6(n-1) \times 6(n-1)$ by using a priori information about the solution.

There are some other approaches for the computation of Riemann-Hilbert problems, though none are applicable to our particular problem. The conjugation method [19] can be used to solve a nonlinear Riemann-Hilbert problem on the unit circle related to conformal mapping. This has been generalized to multiple circles $[\mathbf{1 8}, \mathbf{2 0}]$, but not to unit intervals or curves like our $\Gamma$. Another approach applicable to smooth closed curves is based on solving integral equations $[\mathbf{1 5}]$. 


\section{Choice of basis}

Each curve $\Gamma_{\kappa}$ is a ray in the complex plane. An oft-used technique from spectral methods, which we employ, is to represent a function defined on a ray by mapping it to function defined on the unit interval. Indeed, we can conformally map the unit interval to $\Gamma_{\kappa}$ using the map

$$
H_{\kappa}(t)=\mathrm{e}^{\mathrm{i} \pi\left(\frac{1}{6}+\frac{\kappa-1}{3}\right)} \frac{t+1}{t-1} .
$$

(The map typically used is $L H_{\kappa}(t)$ for some constant $L[\boldsymbol{6}]$. We fix $L=1$ for simplicity.)

On the unit interval, the natural representation for functions is Chebyshev series.

Definition 2.1 For a fixed integer $n$, define the $n$ Chebyshev-Lobatto points as

$$
\boldsymbol{t}=\left(-1, \cos \pi\left(-1+\frac{1}{n-1}\right), \ldots, \cos \pi\left(1-\frac{1}{n-1}\right), 1\right)^{\top}
$$

We can efficiently represent a function defined on the interval by its values at $\boldsymbol{t}$ :

$$
\boldsymbol{f}=f(\boldsymbol{t}) .
$$

By taking an appropriately scaled discrete cosine transform of $\boldsymbol{f}$, which we denote $\check{\boldsymbol{f}}$, we obtain the Chebyshev polynomial which interpolates $f$ at $\boldsymbol{t}$ :

$$
\boldsymbol{e}(t)^{\top} \boldsymbol{f}=\left(T_{0}(t), \ldots, T_{n-1}(t)\right) \check{\boldsymbol{f}} .
$$

Alternatively, the barycentric formula can be used [5].

Definition 2.2 For a vector $\boldsymbol{x}=\left(x_{1}, \ldots, x_{n}\right)^{\top}$, the notation $\overline{\boldsymbol{x}}$ denotes $\boldsymbol{x}$ with its last entry removed: $\overline{\boldsymbol{x}}=\left(x_{1}, \ldots, x_{n-1}\right)^{\top}$.

Using the map $H_{\kappa}$ and the fact that every function $r$ we consider vanishes at $\infty$, we can thence represent a function $r$ defined on $\Gamma_{\kappa}$ by its values at $n-1$ mapped Chebyshev-Lobatto points:

$$
\overline{\boldsymbol{r}}_{\kappa}=r\left(\overline{\boldsymbol{z}}_{\kappa}\right) \quad \text { for } \quad \overline{\boldsymbol{z}}_{\kappa}=H_{\kappa}(\overline{\boldsymbol{t}})
$$

The values at the points $\boldsymbol{z}_{\kappa}=H_{\kappa}(\boldsymbol{t})$ are $\boldsymbol{r}_{\kappa}=\left(\begin{array}{c}\overline{\boldsymbol{r}}_{\kappa} \\ 0\end{array}\right)$. Then the function

$$
\boldsymbol{e}\left(H_{\kappa}^{-1}(z)\right)^{\top} \boldsymbol{r}_{\kappa}
$$

interpolates $r$ at the points $\boldsymbol{z}_{\kappa}$. This is referred to as a rational Chebyshev interpolant [6]. Thus we can approximate a function $r$ which is smooth along each $\Gamma_{\kappa}$ by the function

$$
\left\{\begin{array}{c}
\boldsymbol{e}\left(H_{1}^{-1}(z)\right)^{\top} \boldsymbol{r}_{1} \quad z \in \Gamma_{1} \\
\vdots \\
\boldsymbol{e}\left(H_{6}^{-1}(z)\right)^{\top} \boldsymbol{r}_{6} \quad z \in \Gamma_{6}
\end{array}\right.
$$


In the notation of Algorithm 1.4 we use the basis whose elements are one at a point in $\boldsymbol{z}_{\kappa}$ and zero at every other point:

$$
p_{\kappa j}(z)=\boldsymbol{e}\left(H_{\kappa}^{-1}(z)\right)^{\top} \boldsymbol{e}_{j}
$$

Then the coefficients $\boldsymbol{v}_{\kappa}^{(i, j)}$ correspond to function values at the points $\boldsymbol{z}_{\kappa}$.

\section{Computing the Hilbert transform over $\Gamma$}

We now consider the computation of $\mathcal{M}$ over $\Gamma$, which is up to a constant equivalent to the Hilbert transform. We note that

$$
\mathcal{M}_{\Gamma}=\mathcal{M}_{\Gamma_{1}}+\ldots+\mathcal{M}_{\Gamma_{6}}
$$

since $\mathcal{M}_{\Gamma_{\kappa}} f$ is analytic off $\Gamma_{\kappa}$; hence, $\mathcal{M}_{\Gamma_{\kappa}}^{+} f-\mathcal{M}_{\Gamma_{\kappa}}^{-} f=0$ along $\Gamma_{\gamma}$ for $\gamma \neq \kappa$. Therefore we need only consider the computation of

$$
\mathcal{M}_{\Gamma_{1}}, \ldots, \mathcal{M}_{\Gamma_{6}}
$$

at the points $\boldsymbol{z}_{1}, \ldots, \boldsymbol{z}_{6}$. The operator $\mathcal{L}$ uses the \pm limits of its inputs. However, we know for $z \notin \Gamma_{\kappa}$ that

$$
\mathcal{M}_{\Gamma_{\kappa}}^{+} r(z)=\mathcal{M}_{\Gamma_{\kappa}}^{-} r(z)=\mathcal{M}_{\Gamma_{\kappa}} r(z),
$$

since $\mathcal{M}_{\Gamma_{\kappa}} f$ is analytic off $\Gamma_{\kappa}$. On the other hand, for $z \in \Gamma_{\kappa}$ they are not necessarily equal.

We have a map $H_{\kappa}$ from the unit interval to the curve $\Gamma_{\kappa}$. But, similar to the developement in [17], we can write $\mathcal{M}_{\Gamma_{\kappa}}$ in terms of $\mathcal{M}_{(-1,1)}$ using this map: if $f(t)=r\left(H_{\kappa}(t)\right)$ and $\Phi=\mathcal{M}_{(-1,1)} f$, then

$$
\mathcal{M}_{\Gamma_{\kappa}} r(z)=\Phi\left(H_{\kappa}^{-1}(z)\right)-\Phi^{+}(1) .
$$

This is easily confirmed by looking at the behaviour as $z$ approaches $\Gamma_{\kappa}$ from the left and right:

$$
\begin{aligned}
\mathcal{M}_{\Gamma_{\kappa}}^{+} r(z)-\mathcal{M}_{\Gamma_{\kappa}}^{-} r(z) & =\Phi^{+}\left(H_{\kappa}^{-1}(z)\right)-\Phi^{-}\left(H_{\kappa}^{-1}(z)\right)=f\left(H_{\kappa}^{-1}(z)\right)=r(z), \\
\lim _{z \rightarrow \infty} \mathcal{M}_{\Gamma_{\kappa}} r(z) & =\lim _{z \rightarrow \infty} \Phi\left(H_{\kappa}^{-1}(z)\right)-\Phi^{+}(1)=\lim _{t \rightarrow 1} \Phi(t)-\Phi^{+}(1)=0 .
\end{aligned}
$$

Moreover, we have an expression for $\mathcal{M}_{(-1,1)}$ in closed form. This expression is derived by mapping the interval to the unit circle, using the Chebyshev map and its inverses:

Definition 3.1 The Chebyshev map

$$
T(z)=\frac{1}{2}\left(z+\frac{1}{z}\right)
$$

maps the interior and exterior of the unit circle to $\mathbb{C} \backslash[-1,1]$. Thus it has two inverses defined in $\mathbb{C} \backslash[-1,1]$ :

$$
T_{ \pm}^{-1}(t)=t \mp \sqrt{t-1} \sqrt{t+1}
$$


$T_{+}^{-1}$ and $T_{-}^{-1} \operatorname{map} \mathbb{C} \backslash[-1,1]$ to the interior and exterior of the circle, respectively. Since $T_{ \pm}$ each have a branch cut along $[-1,1]$, we need two additional inverses:

$$
T_{\uparrow}^{-1}(t)=t+\mathrm{i} \sqrt{1-t} \sqrt{1+t} \quad \text { and } \quad T_{\downarrow}^{-1}(t)=t-\mathrm{i} \sqrt{1-t} \sqrt{1+t} .
$$

These map $[-1,1]$ to the upper and lower half of the unit circle, respectively, and are analytic along the interval.

Using these maps, we obtain the following formulæ:

Theorem $3.2[17]$ Suppose

$$
f(x)=\sum_{k=0}^{\infty} \check{f}_{k} T_{k}(x) \quad \text { for } \quad x \in[-1,1]
$$

is $\mathbb{C}^{1}[-1,1]$ and its first derivative has bounded variation. Define

$$
\begin{aligned}
& \psi_{0}(z)=\frac{2}{\mathrm{i} \pi}\left\{\begin{array}{ll}
\operatorname{arctanh} z & \text { for }|z|<1 \\
\operatorname{arctanh} \frac{1}{z} & \text { for }|z|>1
\end{array},\right. \\
& \mu_{m}(z)=\sum_{j=1}^{\left.\frac{m+1}{2}\right\rfloor} \frac{z^{2 j-1}}{2 j-1}=\operatorname{arctanh} z-\frac{1}{2} z^{2\left\lfloor\frac{m+1}{2}\right\rfloor+1} \phi\left(z^{2}, 1, \frac{1}{2}+\left\lfloor\frac{m+1}{2}\right\rfloor\right), \\
& \psi_{m}(z)=\frac{2}{\mathrm{i} \pi} \begin{cases}z^{m+2\left\lfloor\frac{-m}{2}\right\rfloor+1} \phi\left(z^{2}, 1, \frac{1}{2}+\left\lfloor\frac{-m}{2}\right\rfloor\right) & \text { for } m<0 \\
z^{m-2\left\lfloor\frac{m+1}{2}\right\rfloor-1} \phi\left(z^{-2}, 1, \frac{1}{2}+\left\lfloor\frac{m+1}{2}\right\rfloor\right) & \text { for } m>0\end{cases}
\end{aligned}
$$

where $\phi$ is the Lerch transcendental function [4]. Then

$$
\begin{aligned}
& \mathcal{M}_{(-1,1)} f(t)=-\frac{1}{4} \sum_{k=0}^{\infty} \check{f}_{k}\left[\psi_{k}\left(T_{+}^{-1}(t)\right)+\psi_{k}\left(T_{-}^{-1}(t)\right)+\psi_{-k}\left(T_{+}^{-1}(t)\right)+\psi_{-k}\left(T_{-}^{-1}(t)\right)\right] \\
& \mathcal{M}_{(-1,1)} f(t) \underset{t \rightarrow-1}{\sim}-\frac{1}{2 \mathrm{i} \pi} f(-1)[\log (-t-1)-\log 2] \\
& \quad+\frac{1}{\mathrm{i} \pi} \sum_{k=0}^{\infty} \check{f}_{k}(-1)^{k}\left[\mu_{k-1}(-1)+\mu_{k}(-1)\right] \\
& \mathcal{M}_{(-1,1)} f(t) \underset{t \rightarrow 1}{\sim} \frac{1}{2 \mathrm{i} \pi} f(1)[\log (t-1)-\log 2]+\frac{1}{\mathrm{i} \pi} \sum_{k=0}^{\infty} \check{f}_{k}\left[\mu_{k-1}(1)+\mu_{k}(1)\right]
\end{aligned}
$$

and, for $t \in(-1,1)$,

$$
\begin{aligned}
& \mathcal{M}_{(-1,1)}^{+} f(t)=-\frac{1}{4} \sum_{k=0}^{\infty} \check{f}_{k}\left[\psi_{k}^{+}\left(T_{\downarrow}^{-1}(t)\right)+\psi_{k}^{-}\left(T_{\uparrow}^{-1}(t)\right)+\psi_{-k}^{+}\left(T_{\downarrow}^{-1}(t)\right)+\psi_{-k}^{-}\left(T_{\uparrow}^{-1}(t)\right)\right], \\
& \mathcal{M}_{(-1,1)}^{-} f(t)=-\frac{1}{4} \sum_{k=0}^{\infty} \check{f}_{k}\left[\psi_{k}^{+}\left(T_{\uparrow}^{-1}(t)\right)+\psi_{k}^{-}\left(T_{\downarrow}^{-1}(t)\right)+\psi_{-k}^{+}\left(T_{\uparrow}^{-1}(t)\right)+\psi_{-k}^{-}\left(T_{\downarrow}^{-1}(t)\right)\right] .
\end{aligned}
$$


Remark: One approach to computing $\psi_{m}$ is to use the method developed in [3], or the built-in Mathematica routine. These approaches are currently slower and less accurate than using high precision arithmetic with the fact that $\psi_{m}$ is the arctanh function plus a polynomial in $z$, cf. [17], which is derived using the Taylor series of arctanh at zero. We use this to compute its value to machine precision.

Using this formula, we can derive an expression for $\mathcal{M}_{\Gamma_{\kappa}}$. We note that when $r(z)=$ $\mathcal{O}\left(z^{-1}\right)$ as $z \rightarrow \infty, f(t)=r\left(H_{\kappa}(t)\right)=\mathcal{O}(t-1)$ as $t \rightarrow 1$. Therefore the unbounded term in (3.2) vanishes, and we obtain

$$
\mathcal{M}_{\Gamma_{\kappa}} r(z)=\mathcal{M}_{(-1,1)} f\left(H_{\kappa}^{-1}(z)\right)-\frac{1}{\mathrm{i} \pi} \sum_{k=0}^{\infty} \check{f}_{\kappa}\left[\mu_{k-1}(1)+\mu_{k}(1)\right] .
$$

Now consider the function

$$
\mathcal{M}_{\Gamma} r(z)=\mathcal{M}_{\Gamma_{1}} r(z)+\ldots+\mathcal{M}_{\Gamma_{6}} r(z) .
$$

Denote the restriction of $r$ to $\Gamma_{\kappa}$ as $r_{\kappa}$, where $r_{\kappa}(0)$ is the limit of $r$ as $z \rightarrow 0$ along $\Gamma_{\kappa}$. Each term $\mathcal{M}_{\Gamma_{\kappa}} r_{\kappa}$ blows up at zero, however, to solve the Riemann-Hilbert problem we need the solution to be bounded at zero. From (3.1) we can determine the behaviour of the blow up at zero:

$$
\begin{aligned}
\mathcal{M}_{\Gamma_{\kappa}} r_{\kappa}(z) \underset{z \rightarrow 0}{\sim} & -\frac{1}{2 \mathrm{i} \pi} r_{\kappa}\left(H_{\kappa}(-1)\right)\left[\log \left(-H_{\kappa}^{-1}(z)-1\right)-\log 2\right] \\
& +\frac{1}{\mathrm{i} \pi} \sum_{k=0}^{\infty} \check{f}_{k}\left\{(-1)^{k}\left[\mu_{k-1}(-1)+\mu_{k}(-1)\right]-\mu_{k-1}(1)-\mu_{k}(1)\right\} \\
\sim & -\frac{1}{2 \mathrm{i} \pi} r_{\kappa}(0) \log \left(-2 \mathrm{e}^{-\mathrm{i}\left[\frac{\pi}{6}+\frac{\pi}{3}(\kappa-1)\right]} z\right)+C \\
= & -\frac{1}{2 \mathrm{i} \pi} r_{\kappa}(0)\left(\log |2 z|+\mathrm{i} \arg \left[-\mathrm{e}^{-\mathrm{i}\left[\frac{\pi}{6}+\frac{\pi}{3}(\kappa-1)\right]} z\right]\right)+C \\
\sim & -\frac{1}{2 \mathrm{i} \pi} r_{\kappa}(0) \log |2 z| .
\end{aligned}
$$

Thus, if $r_{1}(z)+\cdots+r_{6}(z)=\mathcal{O}(z)$ as $z \rightarrow 0$, then the unbounded contribution of $\mathcal{M}_{\Gamma} r(z)=$ $\mathcal{M}_{\Gamma_{1}} r_{1}(z)+\cdots+\mathcal{M}_{\Gamma_{6}} r_{6}(z)$ is

$$
\lim _{z \rightarrow 0}-\frac{1}{2 \mathrm{i} \pi}\left(r_{1}(z)+\cdots+r_{6}(z)\right) \log |2 z|=0 .
$$

We now consider again the case where a function $r$ is represented on $\Gamma_{1}$ by $\overline{\boldsymbol{r}}_{1}=r_{1}\left(\overline{\boldsymbol{z}}_{1}\right)$. We want to construct an $(n-1) \times(n-1)$ matrix $M^{+}$that maps $\overline{\boldsymbol{r}}_{1}$ to the values of $\mathcal{M}_{\Gamma_{1}}^{+} \boldsymbol{e}\left(H_{1}^{-1}(z)\right)^{\top} \boldsymbol{r}_{1}$ at the points $\overline{\boldsymbol{z}}_{1}$. In other words, for $j=1, \ldots, n-1$, we would like

$$
M^{+} \overline{\boldsymbol{e}}_{j} \stackrel{?}{=} \mathcal{M}_{\Gamma_{1}}^{+} \boldsymbol{e}\left(H_{1}^{-1}\left(\overline{\boldsymbol{z}}_{1}\right)\right)^{\top} \boldsymbol{e}_{j} .
$$


The first row of this matrix is unbounded, hence we instead assume that the logarithmic singularity is cancelled. Thus we choose $M^{+}$so that

$$
M^{+} \overline{\boldsymbol{e}}_{j}=\left(\varphi_{0}^{+}(\overline{\boldsymbol{t}}), \ldots, \varphi_{n-1}^{+}(\overline{\boldsymbol{t}})\right) \check{\boldsymbol{e}}_{j}
$$

for

$$
\begin{aligned}
& \varphi_{k}^{+}(t)=- \frac{1}{4}\left[\psi_{k}^{+}\left(T_{\downarrow}^{-1}(t)\right)+\psi_{k}^{-}\left(T_{\uparrow}^{-1}(t)\right)+\psi_{-k}^{+}\left(T_{\downarrow}^{-1}(t)\right)+\psi_{-k}^{-}\left(T_{\uparrow}^{-1}(t)\right)\right] \\
&-\frac{\mu(-1,1)-\mu(0,1)}{\mathrm{i} \pi} \\
& \varphi_{k}^{+}(0)=(-1)^{k}\left[\frac{\log 2}{2 \mathrm{i} \pi}+\frac{1}{2}\right]+\frac{1}{\mathrm{i} \pi}\left\{(-1)^{k}\left[\mu_{k-1}(-1)+\mu_{k}(-1)\right]-\mu_{k-1}(1)-\mu_{k}(1)\right\}
\end{aligned}
$$

Therefore the matrix $M^{+}$can be constructed by computing $\varphi_{0}^{+}(\overline{\boldsymbol{t}}), \ldots, \varphi_{n-1}^{+}(\overline{\boldsymbol{t}})$ and applying the discrete cosine transform on the right. If the unbounded growth is somehow cancelled, then applying $M^{+}$to $\boldsymbol{r}$ is equivalent to computing $\mathcal{M}_{\Gamma_{1}} \boldsymbol{e}\left(H_{1}^{-1}(z)\right)^{\top} \boldsymbol{r}$ at the points $\overline{\boldsymbol{z}}_{1}$. In particular, this is the case when $\boldsymbol{e}_{1}^{\top} \boldsymbol{r}=0$.

Similarly, we can define $M^{-}$via

$$
M^{-} \overline{\boldsymbol{e}}_{j}=\left(\varphi_{0}^{-}(\overline{\boldsymbol{t}}), \ldots, \varphi_{n-1}^{-}(\overline{\boldsymbol{t}})\right) \check{\boldsymbol{e}}_{j}
$$

for

$$
\begin{aligned}
\varphi_{k}^{-}(t)=- & \frac{1}{4}\left[\psi_{k}^{+}\left(T_{\uparrow}^{-1}(t)\right)+\psi_{k}^{-}\left(T_{\downarrow}^{-1}(t)\right)+\psi_{-k}^{+}\left(T_{\uparrow}^{-1}(t)\right)+\psi_{-k}^{-}\left(T_{\downarrow}^{-1}(t)\right)\right] \\
& -\frac{\mu(-1,1)-\mu(0,1)}{\mathrm{i} \pi} \\
\varphi_{k}^{-}(0)= & (-1)^{k}\left[\frac{\log 2}{2 \mathrm{i} \pi}-\frac{1}{2}\right]+\frac{1}{\mathrm{i} \pi}\left\{(-1)^{k}\left[\mu_{k-1}(-1)+\mu_{k}(-1)\right]-\mu_{k-1}(1)-\mu_{k}(1)\right\}
\end{aligned}
$$

Finally, we define $M_{\gamma}$, corresponding to applying $\mathcal{M}$ along $\Gamma_{\gamma}$ for $\gamma=2, \ldots, 6$, by

$$
M_{\gamma} \overline{\boldsymbol{e}}_{j}=\left(\varphi_{0}\left(H_{1}^{-1}\left(\overline{\boldsymbol{z}}_{\gamma}\right)\right), \ldots, \varphi_{n-1}\left(H_{1}^{-1}\left(\overline{\boldsymbol{z}}_{\gamma}\right)\right)\right) \check{\boldsymbol{e}}_{j}
$$

where

$$
\begin{aligned}
\varphi_{k}(t)=- & \frac{1}{4}\left[\psi_{k}\left(T_{+}^{-1}(t)\right)+\psi_{k}\left(T_{-}^{-1}(t)\right)+\psi_{-k}\left(T_{+}^{-1}(t)\right)+\psi_{-k}\left(T_{-}^{-1}(t)\right)\right] \\
& -\frac{\mu(-1,1)-\mu(0,1)}{\mathrm{i} \pi} \\
\varphi_{k}(0)=(-1)^{k}\left[\frac{\log 2}{2 \mathrm{i} \pi}+\frac{2}{3}-\frac{\gamma}{6}\right] & \\
& +\frac{1}{\mathrm{i} \pi}(-1)^{k}\left[\mu_{k-1}(-1)+\mu_{k}(-1)-\mu_{k-1}(1)+\mu_{k}(1)\right]
\end{aligned}
$$


This is related to computing $\mathcal{M}_{\Gamma_{1}}$ along $\Gamma_{\gamma}$.

Ostensibly we would need to redo these calculations for each $\Gamma_{\kappa}$ to compute $\mathcal{M}_{\Gamma_{\kappa}}$. However, computing $\mathcal{M}$ is invariant under rotation. Therefore (ignoring the unboundedness at zero)

$$
\begin{aligned}
M^{ \pm} \overline{\boldsymbol{r}}_{\kappa} & =\mathcal{M}_{\Gamma_{\kappa}}^{ \pm} \boldsymbol{e}\left(H_{\kappa}^{-1}\left(\overline{\boldsymbol{z}}_{\kappa}\right)\right)^{\top} \boldsymbol{r}_{\kappa}, \\
M_{\gamma-\kappa+1 \bmod 6} \overline{\boldsymbol{r}}_{\kappa} & =\mathcal{M}_{\Gamma_{\kappa}}^{ \pm} \boldsymbol{e}\left(H_{\kappa}^{-1}\left(\overline{\boldsymbol{z}}_{\gamma}\right)\right)^{\top} \boldsymbol{r}_{\kappa} .
\end{aligned}
$$

If $\boldsymbol{e}_{1}^{\top} \boldsymbol{r}_{1}+\cdots+\boldsymbol{e}_{1}^{\top} \boldsymbol{r}_{6}$, then we obtain

$$
\begin{aligned}
\mathcal{M}_{\Gamma}^{ \pm} r\left(\overline{\boldsymbol{z}}_{1}\right) & \approx \mathcal{M}_{\Gamma_{1}}^{ \pm} \boldsymbol{e}\left(\overline{\boldsymbol{z}}_{1}\right)^{\top} \boldsymbol{r}_{1}+\cdots+\mathcal{M}_{\Gamma_{6}} \boldsymbol{e}\left(\overline{\boldsymbol{z}}_{1}\right)^{\top} \boldsymbol{r}_{6} \\
& =M^{ \pm} \overline{\boldsymbol{r}}_{1}+M_{6} \overline{\boldsymbol{r}}_{2}+M_{5} \overline{\boldsymbol{r}}_{3}+\cdots+M_{2} \overline{\boldsymbol{r}}_{6}, \\
\mathcal{M}_{\Gamma}^{ \pm} r\left(\overline{\boldsymbol{z}}_{2}\right) & \approx M_{2} \overline{\boldsymbol{r}}_{1}+M^{ \pm} \overline{\boldsymbol{r}}_{2}+M_{6} \overline{\boldsymbol{r}}_{3}+\cdots+M_{3} \overline{\boldsymbol{r}}_{6}, \\
\vdots & \\
\mathcal{M}_{\Gamma}^{ \pm} r\left(\overline{\boldsymbol{z}}_{6}\right) & \approx M_{6} \overline{\boldsymbol{r}}_{1}+\cdots M_{2} \overline{\boldsymbol{r}}_{5}+M^{ \pm} \overline{\boldsymbol{r}}_{6} .
\end{aligned}
$$

\section{Constructing the least squares system}

We now use the matrices $M^{ \pm}, M_{2}, \ldots, M_{6}$ to construct the linear system (1.5). We first derive some properties of the solution which will allow us to decrease the dimensions of the linear system. Represent $U$ via the definition

$$
U^{(i j)}=\mathcal{M} V^{(i j)}=\sum_{\kappa=1}^{6} \mathcal{M}_{\Gamma_{\kappa}} V_{\kappa}^{(i j)}
$$

Now consider the case where $\gamma$ is odd. Since $\mathcal{M}_{\Gamma_{\kappa}} V_{\kappa}^{(i j)}$ is analytic off $\Gamma_{\kappa}$, and, by definition, $\mathcal{M}_{\Gamma_{\gamma}}^{+} r-\mathcal{M}_{\Gamma_{\gamma}}^{-} r=r$ along $\Gamma_{\gamma}$, we obtain

$$
U^{(i j)+}-U^{(i j)-}=\sum_{\kappa=1}^{6}\left(\mathcal{M}_{\Gamma_{\kappa}}^{+}-\mathcal{M}_{\Gamma_{\kappa}}^{-}\right) V_{\kappa}^{(i j)}=\left(\mathcal{M}_{\Gamma_{\gamma}}^{+}-\mathcal{M}_{\Gamma_{\gamma}}^{-}\right) V_{\gamma}^{(i j)}=V_{\gamma}^{(i j)}
$$

along $\Gamma_{\gamma}$. This implies that

$$
\begin{aligned}
& \mathcal{L} \mathcal{M} V=U^{+}-U^{-} G=\left(\begin{array}{ll}
U^{(11)+} & U^{(12)+} \\
U^{(21)+} & U^{(22)+}
\end{array}\right)-\left(\begin{array}{ll}
U^{(11)-} & U^{(12)-} \\
U^{(21)-} & U^{(22)-}
\end{array}\right)\left(\begin{array}{cc}
1 & \\
s_{\gamma} \mathrm{e}^{8 \mathrm{i} / 3 z^{3}+2 \mathrm{i} x z} & 1
\end{array}\right) \\
& =\left(\begin{array}{ll}
U^{(11)+}-U^{(11)-}-U^{(12)-} s_{\gamma} \mathrm{e}^{8 \mathrm{i} / 3 z^{3}+2 \mathrm{i} x z} & U^{(12)+}-U^{(12)-} \\
U^{(21)+}-U^{(21)-}-U^{(22)-} s_{\gamma} \mathrm{e}^{8 \mathrm{i} / 3 z^{3}+2 \mathrm{i} x z} & U^{(22)+}-U^{(22)-}
\end{array}\right) \\
& =\left(\begin{array}{ll}
V_{\gamma}^{(11)}-s_{\gamma} \mathrm{e}^{8 \mathrm{i} / 3 z^{3}+2 \mathrm{i} x z} \mathcal{M}^{-} V^{(12)} & V_{\gamma}^{(12)} \\
V_{\gamma}^{(21)}-s_{\gamma} \mathrm{e}^{8 \mathrm{i} / 3 z^{3}+2 \mathrm{i} x z} \mathcal{M}^{-} V^{(22)} & V_{\gamma}^{(22)}
\end{array}\right) .
\end{aligned}
$$


On the other hand, the right-hand side is

$$
G-I=\left(\begin{array}{cc}
0 & 0 \\
s_{\gamma} \mathrm{e}^{8 \mathrm{i} / 3 z^{3}+2 \mathrm{i} x z} & 0
\end{array}\right)
$$

Therefore, we obtain trivially that $0=V_{\gamma}^{(12)}=V_{\gamma}^{(22)}$, or in other words, the contribution to $U^{(12)}$ and $U^{(22)}$ from $\Gamma_{\gamma}$ is zero and

$$
\boldsymbol{v}_{\gamma}=\left(\begin{array}{ll}
\boldsymbol{v}_{\gamma}^{(11)} & 0 \\
\boldsymbol{v}_{\gamma}^{(21)} & 0
\end{array}\right)
$$

Similarly, when $\gamma$ is even we obtain

$$
\mathcal{L} \mathcal{M} V=\left(\begin{array}{cc}
V_{\gamma}^{(11)} & V_{\gamma}^{(12)}-s_{2} \mathrm{e}^{-8 \mathrm{i} / 3 z^{3}-2 \mathrm{i} x z} \mathcal{M}^{-} V^{(11)} \\
V_{\gamma}^{(21)} & V_{\gamma}^{(22)}-s_{\gamma} \mathrm{e}^{-8 \mathrm{i} / 3 z^{3}-2 \mathrm{i} x z} \mathcal{M}^{-} V^{(21)}
\end{array}\right)
$$

and a right-hand side of

$$
G-I=\left(\begin{array}{cc}
0 & s_{\gamma} \mathrm{e}^{-8 \mathrm{i} / 3 z^{3}-2 \mathrm{i} x z} \\
0 & 0
\end{array}\right)
$$

Therefore

$$
\boldsymbol{v}_{\gamma}=\left(\begin{array}{cc}
0 & \boldsymbol{v}_{\gamma}^{(12)} \\
0 & \boldsymbol{v}_{\gamma}^{(22)}
\end{array}\right)
$$

In other words, $V_{\gamma}^{(11)}$ and $V_{\gamma}^{(21)}$ are only nonzero for odd $\gamma, V_{\gamma}^{(21)}$ and $V_{\gamma}^{(22)}$ are only nonzero for even $\gamma$.

We now have all the tools necessary to convert the Riemann-Hilbert problem into a linear system, which can in fact be split into two independent $6(n-1) \times 6(n-1)$ linear systems. The first system is for the unknowns associated with the (11) and (12) entries of $V:$

$$
\begin{aligned}
& \overline{\boldsymbol{v}}_{1}^{(11)}-s_{1} \operatorname{diag}\left(\mathrm{e}^{8 \mathrm{i} / 3 \bar{z}_{1}^{3}+2 \mathrm{i} x \bar{z}_{1}}\right)\left[M_{6} \overline{\boldsymbol{v}}_{2}^{(12)}+M_{4} \overline{\boldsymbol{v}}_{4}^{(12)}+M_{2} \overline{\boldsymbol{v}}_{6}^{(12)}\right]=\mathbf{0}, \\
& \overline{\boldsymbol{v}}_{2}^{(12)}-s_{2} \operatorname{diag}\left(\mathrm{e}^{-8 \mathrm{i} / 3 \bar{z}_{2}^{3}-2 \mathrm{i} x \bar{z}_{2}}\right)\left[M_{2} \overline{\boldsymbol{v}}_{1}^{(11)}+M_{6} \overline{\boldsymbol{v}}_{3}^{(11)}+M_{4} \overline{\boldsymbol{v}}_{5}^{(11)}\right]=s_{2} \mathrm{e}^{-8 \mathrm{i} / 3 \bar{z}_{2}^{3}-2 \mathrm{i} x \bar{z}_{2}}, \\
& \overline{\boldsymbol{v}}_{3}^{(11)}-s_{3} \operatorname{diag}\left(\mathrm{e}^{8 \mathrm{i} / 3 \bar{z}_{3}^{3}+2 \mathrm{i} x \bar{z}_{3}}\right)\left[M_{2} \overline{\boldsymbol{v}}_{2}^{(12)}+M_{6} \overline{\boldsymbol{v}}_{4}^{(12)}+M_{4} \overline{\boldsymbol{v}}_{6}^{(12)}\right]=\mathbf{0}, \\
& \overline{\boldsymbol{v}}_{4}^{(12)}+s_{1} \operatorname{diag}\left(\mathrm{e}^{-8 \mathrm{i} / 3 \bar{z}_{4}^{3}-2 \mathrm{i} x \overline{\boldsymbol{z}}_{4}}\right)\left[M_{4} \overline{\boldsymbol{v}}_{1}^{(11)}+M_{2} \overline{\boldsymbol{v}}_{3}^{(11)}+M_{6} \overline{\boldsymbol{v}}_{5}^{(11)}\right]=-s_{1} \mathrm{e}^{-8 \mathrm{i} / 3 \overline{\boldsymbol{z}}_{4}^{3}-2 \mathrm{i} x \overline{\boldsymbol{z}}_{4}}, \\
& \overline{\boldsymbol{v}}_{5}^{(11)}+s_{2} \operatorname{diag}\left(\mathrm{e}^{8 \mathrm{i} / 3 \bar{z}_{5}^{3}+2 \mathrm{i} x \bar{z}_{5}}\right)\left[M_{4} \overline{\boldsymbol{v}}_{2}^{(12)}+M_{2} \overline{\boldsymbol{v}}_{4}^{(12)}+M_{6} \overline{\boldsymbol{v}}_{6}^{(12)}\right]=\mathbf{0}, \\
& \overline{\boldsymbol{v}}_{6}^{(12)}+s_{3} \operatorname{diag}\left(\mathrm{e}^{-8 \mathrm{i} / 3 \overline{\boldsymbol{z}}_{6}^{3}-2 \mathrm{i} x \overline{\boldsymbol{z}}_{6}}\right)\left[M_{6} \overline{\boldsymbol{v}}_{1}^{(11)}+M_{4} \overline{\boldsymbol{v}}_{3}^{(11)}+M_{2} \overline{\boldsymbol{v}}_{5}^{(11)}\right]=-s_{3} \mathrm{e}^{-8 \mathrm{i} / 3 \overline{\boldsymbol{z}}_{6}^{3}-2 \mathrm{i} x \overline{\boldsymbol{z}}_{6}} \text {. }
\end{aligned}
$$



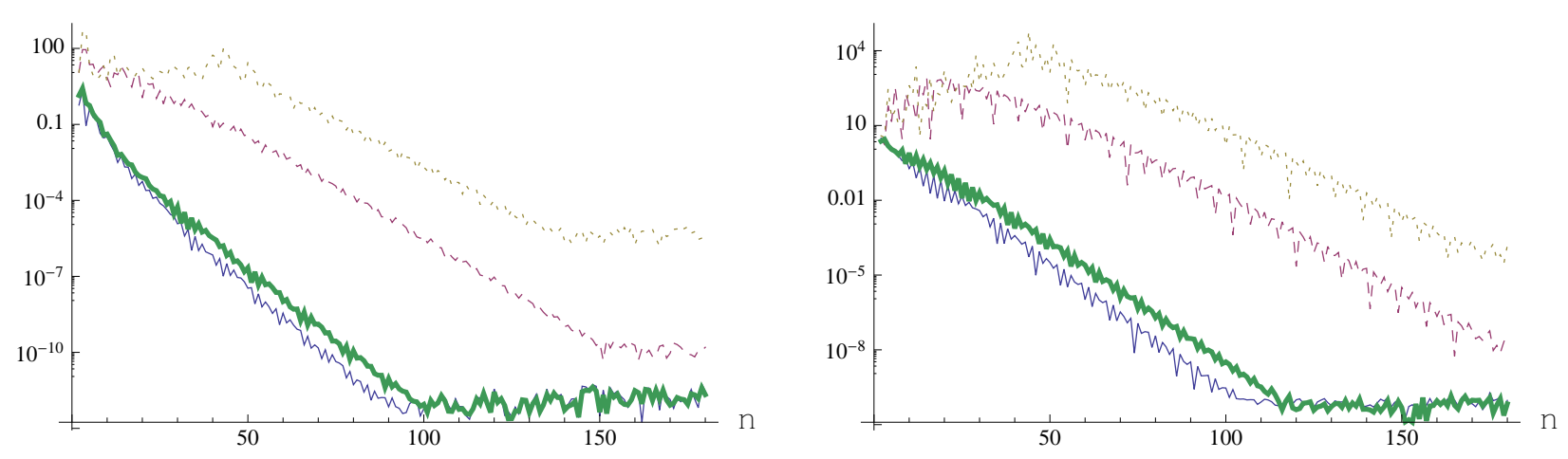

Figure 2: The convergence of the first entry of the solution vectors (first graph) and the approximation of $u_{n}$ compared to $u_{200}$ (right graph) for $x=0$ (plain), 6 (dashed), 8 (dotted) and i (thick).

The second system is for the unknowns associated with the (21) and (22) entries:

$$
\begin{aligned}
\overline{\boldsymbol{v}}_{1}^{(21)}-s_{1} \operatorname{diag}\left(\mathrm{e}^{8 \mathrm{i} / 3 \bar{z}_{1}^{3}+2 \mathrm{i} x \overline{\boldsymbol{z}}_{1}}\right)\left[M_{6} \overline{\boldsymbol{v}}_{2}^{(22)}+M_{4} \overline{\boldsymbol{v}}_{4}^{(22)}+M_{2} \overline{\boldsymbol{v}}_{6}^{(22)}\right]=s_{1} \mathrm{e}^{8 \mathrm{i} / 3 \bar{z}_{1}^{3}+2 \mathrm{i} x \bar{z}_{1}}, \\
\overline{\boldsymbol{v}}_{2}^{(22)}-s_{2} \operatorname{diag}\left(\mathrm{e}^{-8 \mathrm{i} / 3 \bar{z}_{2}^{3}-2 \mathrm{i} x \bar{z}_{2}}\right)\left[M_{2} \overline{\boldsymbol{v}}_{1}^{(21)}+M_{6} \overline{\boldsymbol{v}}_{3}^{(21)}+M_{4} \overline{\boldsymbol{v}}_{5}^{(21)}\right]=\mathbf{0} \\
\overline{\boldsymbol{v}}_{3}^{(21)}-s_{3} \operatorname{diag}\left(\mathrm{e}^{8 \mathrm{i} / 3 \bar{z}_{3}^{3}+2 \mathrm{i} x \bar{z}_{3}}\right)\left[M_{2} \overline{\boldsymbol{v}}_{2}^{(22)}+M_{6} \overline{\boldsymbol{v}}_{4}^{(22)}+M_{4} \overline{\boldsymbol{v}}_{6}^{(22)}\right]=s_{3} \mathrm{e}^{8 \mathrm{i} / 3 \bar{z}_{3}^{3}+2 \mathrm{i} x \bar{z}_{3}}, \\
\overline{\boldsymbol{v}}_{4}^{(22)}+s_{1} \operatorname{diag}\left(\mathrm{e}^{\left.-8 \mathrm{i} / 3 \bar{z}_{4}^{3}-2 \mathrm{i} x \overline{\boldsymbol{z}}_{4}\right)}\left[M_{4} \overline{\boldsymbol{v}}_{1}^{(21)}+M_{2} \overline{\boldsymbol{v}}_{3}^{(21)}+M_{6} \overline{\boldsymbol{v}}_{5}^{(21)}\right]=\mathbf{0},\right. \\
\overline{\boldsymbol{v}}_{5}^{(21)}+s_{2} \operatorname{diag}\left(\mathrm{e}^{\left.8 \mathrm{i} / 3 \bar{z}_{5}^{3}+2 \mathrm{i} x \overline{\boldsymbol{z}}_{5}\right)}\left[M_{4} \overline{\boldsymbol{v}}_{2}^{(22)}+M_{2} \overline{\boldsymbol{v}}_{4}^{(22)}+M_{6} \overline{\boldsymbol{v}}_{6}^{(22)}\right]=-s_{2} \mathrm{e}^{8 \mathrm{i} / 3 \bar{z}_{5}^{3}+2 \mathrm{i} x \overline{\boldsymbol{z}}_{5}},\right. \\
\overline{\boldsymbol{v}}_{6}^{(22)}+s_{3} \operatorname{diag}\left(\mathrm{e}^{\left.-8 \mathrm{i} / 3 \bar{z}_{6}^{3}-2 \mathrm{i} x \overline{\boldsymbol{z}}_{6}\right)}\left[M_{6} \overline{\boldsymbol{v}}_{1}^{(21)}+M_{4} \overline{\boldsymbol{v}}_{3}^{(21)}+M_{2} \overline{\boldsymbol{v}}_{5}^{(21)}\right]=\mathbf{0 .}\right.
\end{aligned}
$$

Though we have described how to construct $M_{ \pm}$and $M_{\gamma}$ for odd $\gamma$, we only require the computation of $M_{2}, M_{4}$ and $M_{6}$. This simplification will not necessarily be possible for other Riemann-Hilbert problems.

For the conversion from the Riemann-Hilbert problem to the value of the solution to Painlevé II at $x$, we require the (12) entry of $\Phi$, which is also the (12) entry of $U$. Thus we need only solve the first linear system. Assuming the linear system is nonsingular, we denote the solution vectors for a given $n$ as $\boldsymbol{v}_{\kappa}^{(i j), n}$. and the approximation of $V$ and $U$ as

$$
\begin{aligned}
V_{\kappa}^{n}(z) & =\left(\begin{array}{ll}
\boldsymbol{e}\left(H_{\kappa}^{-1}(z)\right)^{\top} \boldsymbol{v}_{\kappa}^{(11), n} & \boldsymbol{e}\left(H_{\kappa}^{-1}(z)\right)^{\top} \boldsymbol{v}_{\kappa}^{(12), n} \\
\boldsymbol{e}\left(H_{\kappa}^{-1}(z)\right)^{\top} \boldsymbol{v}_{\kappa}^{(21), n} & \boldsymbol{e}\left(H_{\kappa}^{-1}(z)\right)^{\top} \boldsymbol{v}_{\kappa}^{(22), n}
\end{array}\right) \quad \text { and } \\
U^{n}(z) & =\mathcal{M}_{\Gamma_{1}} V_{1}^{n}(z)+\cdots+\mathcal{M}_{\Gamma_{6}} V_{6}^{n}(z) .
\end{aligned}
$$

As an example, consider the choice of constants $\left(s_{1}, s_{2}, s_{3}\right)=(1+\mathrm{i},-2,1-\mathrm{i})$. Since $s_{1}=\bar{s}_{3}$, we know that the corresponding solution to Painlevé II is real on the real axis [10]. 
To demonstrate the rate of convergence, in Figure 2 we compare the first entry of solution vectors (which corresponds to the value at zero along each $\Gamma_{\kappa}$ ) for consecutive choices of $n$ :

$$
\left\|\left(\begin{array}{l}
\boldsymbol{e}_{1}^{\top}\left(\boldsymbol{v}_{1}^{(11), n}-\boldsymbol{v}_{1}^{(11), n+1}\right) \\
\boldsymbol{e}_{1}^{\top}\left(\boldsymbol{v}_{3}^{(11), n}-\boldsymbol{v}_{3}^{(11), n+1}\right) \\
\boldsymbol{e}_{1}^{\top}\left(\boldsymbol{v}_{5}^{(11), n}-\boldsymbol{v}_{1}^{(11), n+1}\right) \\
\boldsymbol{e}_{1}^{\top}\left(\boldsymbol{v}_{2}^{(12), n}-\boldsymbol{v}_{2}^{(12), n+1}\right) \\
\boldsymbol{e}_{1}^{\top}\left(\boldsymbol{v}_{4}^{(12), n}-\boldsymbol{v}_{4}^{(12), n+1}\right) \\
\boldsymbol{e}_{1}^{\top}\left(\boldsymbol{v}_{6}^{(12), n}-\boldsymbol{v}_{6}^{(12), n+1}\right)
\end{array}\right)\right\|_{\infty}
$$

As can be seen, these values converges spectrally fast, including for complex $x$, though the rate of convergence and stability degenerates as $x$ becomes large.

Properties of the solution

In the previous example, the linear system was always nonsingular. We cannot expect that this is always the case, as if $x$ corresponds to a pole of the solution $u(x)$, then the corresponding Riemann-Hilbert problem itself is not solvable. We do, however know the following:

Theorem 4.1 The two linear systems are solvable for sufficiently small $\left(s_{1}, s_{2}, s_{3}\right)$.

Proof: When $\left(s_{1}, s_{2}, s_{3}\right)=(0,0,0)$, the matrix associated with each linear system is simply an identity operator. Thus continuity of eigenvalues proves the result.

Q.E.D.

In the construction of $M_{\kappa}$, we determined the value at zero by assuming the solution was bounded. In the following theorem, we demonstrate that, subject to a second constraint, this assumption is valid.

Theorem 4.2 Suppose that both linear systems are nonsingular and that

$$
s_{1} s_{3}-s_{1} s_{2}-s_{2} s_{3} \neq 9 .
$$

Then $U^{n}(z)$ is analytic everywhere off $\Gamma$ and bounded at zero.

Proof:

We focus on the (11) and (12) entries, as the proof for the other two entries is equivalent. The first part of the theorem follows since $\mathcal{M}_{\Gamma_{k}}$ is analytic off $\Gamma_{k}$. The second part will result if we can demonstrate that the first entries sum to zero, cf. (3.3):

$$
\Sigma=\mathbf{0} \quad \text { for } \quad \Sigma=\left(\boldsymbol{e}_{1}^{\top}\left[\boldsymbol{v}_{1}^{(11)}+\boldsymbol{v}_{3}^{(11)}+\boldsymbol{v}_{5}^{(11)}\right] \quad \boldsymbol{e}_{1}^{\top}\left[\boldsymbol{v}_{2}^{(12)}+\boldsymbol{v}_{4}^{(12)}+\boldsymbol{v}_{6}^{(12)}\right]\right) .
$$


Define

$$
\begin{aligned}
& \boldsymbol{\Phi}_{1}^{ \pm}=\left(\overline{\boldsymbol{e}}_{1}^{\top}\left(M^{ \pm} \overline{\boldsymbol{v}}_{1}^{(11)}+M_{5} \overline{\boldsymbol{v}}_{3}^{(11)}+M_{3} \overline{\boldsymbol{v}}_{5}^{(11)}\right)+1 \quad \overline{\boldsymbol{e}}_{1}^{\top}\left(M_{6} \overline{\boldsymbol{v}}_{2}^{(12)}+M_{4} \overline{\boldsymbol{v}}_{4}^{(12)}+M_{2} \overline{\boldsymbol{v}}_{6}^{(12)}\right)\right) \\
& \boldsymbol{\Phi}_{2}^{ \pm}=\left(\overline{\boldsymbol{e}}_{1}^{\top}\left(M_{2} \overline{\boldsymbol{v}}_{1}^{(21)}+M_{6} \overline{\boldsymbol{v}}_{3}^{(21)}+M_{4} \overline{\boldsymbol{v}}_{5}^{(21)}\right)+1 \quad \overline{\boldsymbol{e}}_{1}^{\top}\left(M^{ \pm} \overline{\boldsymbol{v}}_{2}^{(12)}+M_{5} \overline{\boldsymbol{v}}_{4}^{(12)}+M_{3} \overline{\boldsymbol{v}}_{6}^{(12)}\right)\right) \\
& \boldsymbol{\Phi}_{6}^{ \pm}=\left(\overline{\boldsymbol{e}}_{1}^{\top}\left(M_{6} \overline{\boldsymbol{v}}_{1}^{(21)}+M_{4} \overline{\boldsymbol{v}}_{3}^{(21)}+M_{2} \overline{\boldsymbol{v}}_{5}^{(21)}\right)+1 \quad \overline{\boldsymbol{e}}_{1}^{\top}\left(M_{5} \overline{\boldsymbol{v}}_{2}^{(12)}+M_{3} \overline{\boldsymbol{v}}_{4}^{(12)}+M^{ \pm} \overline{\boldsymbol{v}}_{6}^{(12)}\right)\right)
\end{aligned}
$$

We assert that

$$
\boldsymbol{\Phi}_{\kappa}^{+}=\boldsymbol{\Phi}_{\kappa+1}^{-}+\frac{\Sigma}{6}
$$

We first note that, for $j=2, \ldots, n-1$,

$$
\overline{\boldsymbol{e}}_{1}^{\top} M^{+} \overline{\boldsymbol{e}}_{j}=\overline{\boldsymbol{e}}_{1}^{\top} M^{-} \overline{\boldsymbol{e}}_{j}=\overline{\boldsymbol{e}}_{1}^{\top} M_{2} \overline{\boldsymbol{e}}_{j}=\cdots=\overline{\boldsymbol{e}}_{1}^{\top} M_{6} \overline{\boldsymbol{e}}_{j}
$$

This follows since $\boldsymbol{e}\left(H_{\kappa}^{-1}(z)\right)^{\top} \boldsymbol{e}_{j}$ vanishes at zero, hence the logarithmic term of

$$
\mathcal{M} \boldsymbol{e}\left(H_{\kappa}^{-1}(z)\right)^{\top} \boldsymbol{e}_{j}
$$

is cancelled and it is continuous at zero. Therefore, for some constant vector $C$ and constants $v_{1}=\boldsymbol{e}_{1}^{\top} \boldsymbol{v}_{1}^{(11)}, \ldots, v_{6}=\boldsymbol{e}_{1}^{\top} \boldsymbol{v}_{6}^{(12)}$ we can write

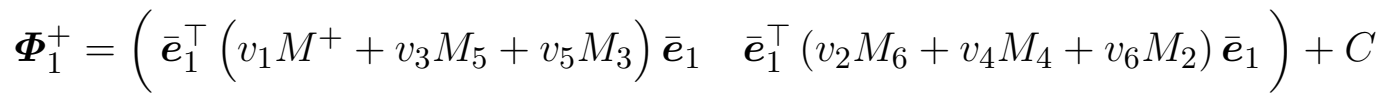

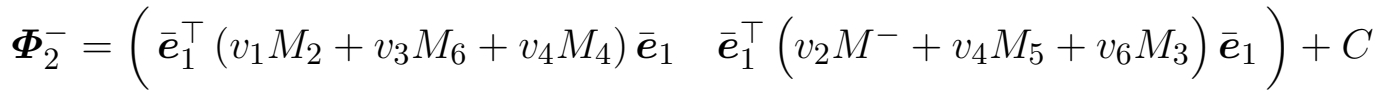

From the definition of $\varphi$ and the fact that $\left(1,-1, \ldots,(-1)^{n}\right) \check{\boldsymbol{e}}_{1}=1$, we find for some constant $D$ that

$$
\begin{aligned}
& \overline{\boldsymbol{e}}_{1}^{\top} M^{+} \overline{\boldsymbol{e}}_{1}=\left(\varphi_{0}^{+}(0), \ldots, \varphi_{n-1}^{+}(0)\right) \check{\boldsymbol{e}}_{1}=\frac{1}{2}+D, \\
& \overline{\boldsymbol{e}}_{1}^{\top} M^{-} \overline{\boldsymbol{e}}_{1}=\left(\varphi_{0}^{-}(0), \ldots, \varphi_{n-1}^{-}(0)\right) \check{\boldsymbol{e}}_{1}=-\frac{1}{2}+D, \\
& \overline{\boldsymbol{e}}_{1}^{\top} M_{\gamma} \overline{\boldsymbol{e}}_{1}=\left(\varphi_{0}(0), \ldots, \varphi_{n-1}(0)\right) \check{\boldsymbol{e}}_{1}=\frac{2}{3}-\frac{\gamma}{6}+D .
\end{aligned}
$$

Thus we get:

$$
\begin{aligned}
\boldsymbol{\Phi}_{1}^{+}-\boldsymbol{\Phi}_{2}^{-} & =\left(\frac{v_{1}}{2}-\frac{v_{1}}{3}-\frac{v_{3}}{6}+\frac{v_{3}}{3}+\frac{v_{5}}{6},-\frac{v_{2}}{3}+\frac{v_{2}}{2}+\frac{v_{4}}{6}+\frac{v_{6}}{3}-\frac{v_{6}}{6}\right) \\
& =\frac{1}{6}\left(v_{1}+v_{3}+v_{5}, v_{2}+v_{4}+v_{6}\right)=\frac{\Sigma}{6} .
\end{aligned}
$$

Similar manipulations prove the identity along the other contours. 
By the design of the linear system, we also know that

$$
\boldsymbol{\Phi}_{\kappa}^{+}=\boldsymbol{\Phi}_{\kappa}^{-} S_{\kappa}
$$

for

$$
S_{1}=\left(\begin{array}{cc}
1 & 0 \\
s_{1} & 1
\end{array}\right), \cdots, S_{6}=\left(\begin{array}{cc}
1 & s_{6} \\
0 & 1
\end{array}\right) .
$$

And, from the analytical development [12], we know that

$$
S_{1} \cdots S_{6}=I
$$

Therefore we obtain

$$
\begin{aligned}
\boldsymbol{\Phi}_{1}^{-} & =\boldsymbol{\Phi}_{1}^{-} S_{1} \cdots S_{6}=\boldsymbol{\Phi}_{1}^{+} S_{2} \cdots S_{6}=\Sigma S_{2} \cdots S_{6}+\boldsymbol{\Phi}_{2}^{-} S_{2} \cdots S_{6} \\
& =\Sigma\left(S_{2} \cdots S_{6}+S_{3} \cdots S_{6}+\cdots+S_{6}+I\right)+\boldsymbol{\Phi}_{1}^{-} .
\end{aligned}
$$

Thus, unless

$$
S_{2} \cdots S_{6}+S_{3} \cdots S_{6}+\cdots+S_{6}+I
$$

happens to be singular, we know that $\Sigma=0$. The determinant of this matrix is

$$
36+4 s_{1} s_{2}-4 s_{1} s_{3}+4 s_{2} s_{3} .
$$

Q.E.D.

The condition that

$$
s_{1} s_{3}-s_{1} s_{2}-s_{2} s_{3} \neq 9
$$

might also be necessary. For example, when $\left(s_{1}, s_{2}, s_{3}\right)=(1,-2-\mathrm{i}, 2-\mathrm{i})$ the linear system itself is singular, though the right-hand side still lies in the span of the matrix. In other words, the kernel of the matrix is nontrivial, and choosing the wrong element of the kernel can cause the solution to not cancel at zero. In this case, the problem can be rectified by imposing the additional conditions

$$
0=\boldsymbol{e}_{1}^{\top}\left[\boldsymbol{v}_{1}^{(11)}+\boldsymbol{v}_{3}^{(11)}+\boldsymbol{v}_{5}^{(11)}\right]=\boldsymbol{e}_{1}^{\top}\left[\boldsymbol{v}_{2}^{(12)}+\boldsymbol{v}_{4}^{(12)}+\boldsymbol{v}_{6}^{(12)}\right],
$$

so that the linear system (now rectangular) is of full rank. It might be possible to show that the system with these additional conditions always has a solution. We leave this problem open.

\section{Converting the solution to the Riemann-Hilbert problem to the solution of Painlevé II}

We have described a method for computing the solution of the Riemann-Hilbert problem associated with the homogeneous Painlevé II equation. Now we want to compute

$$
u(x)=\lim _{z \rightarrow x} \Phi^{(12)}(x ; z)=\lim _{z \rightarrow x} U^{(12)}(x ; z)=\lim _{z \rightarrow x} \mathcal{M} V^{(12)}(x ; z) .
$$



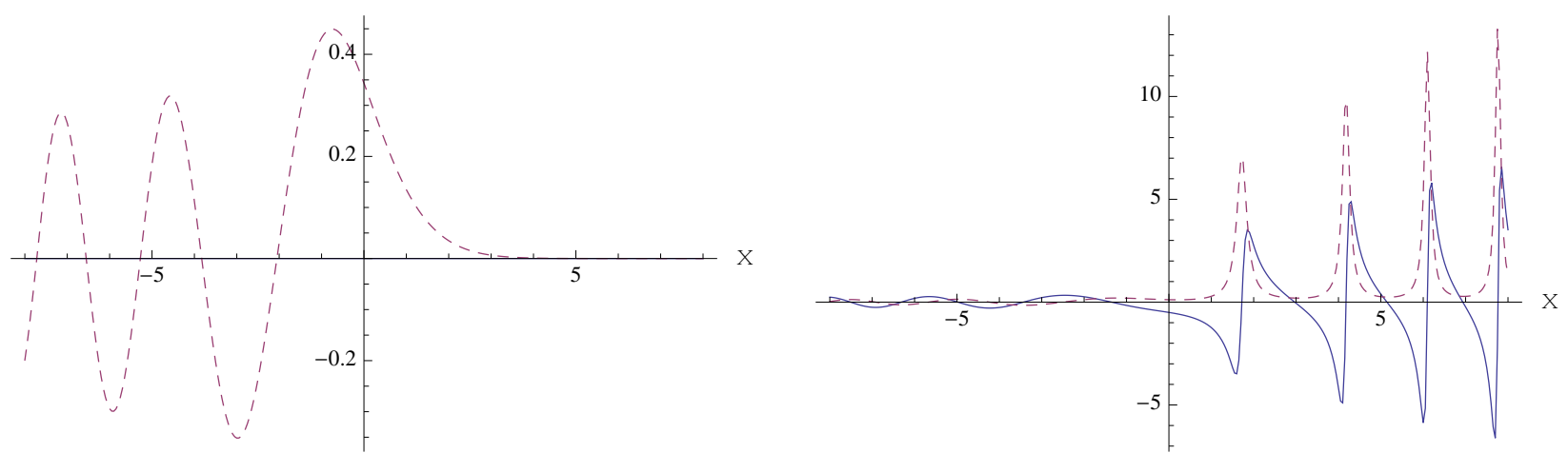

Figure 3: The real (plain) and imaginary (dashed) parts of $u_{150}(x)$ with $\left(s_{1}, s_{2}, s_{3}\right)=(1,0,-1)$ (left) and $\left(s_{1}, s_{2}, s_{3}\right)=(1,2,1 / 3)$ (right).

Since $G(z) \rightarrow I$ as $z \rightarrow \infty$, we know that the direction with which we take this limit is immaterial. Thus we choose $z$ along $\Gamma_{1}$ so as to reuse the matrices $M_{2}, M_{4}$ and $M_{6}$. We can determine approximate values of $\mathcal{M V}$ using:

$$
\mathcal{M} V^{(12)}\left(\overline{\boldsymbol{z}}_{1}\right) \approx \overline{\boldsymbol{u}}=M_{6} \overline{\boldsymbol{v}}_{2}^{(12)}+M_{4} \overline{\boldsymbol{v}}_{4}^{(12)}+M_{2} \overline{\boldsymbol{v}}_{6}^{(12)}
$$

Now we want to find $\lim _{z \rightarrow \infty} z \boldsymbol{e}\left(H_{1}^{-1}(z)\right)^{\top} \boldsymbol{u}$. We make the transformation $z=H_{1}(t)$, so that

$$
\begin{aligned}
u_{n}(x) & =2 \lim _{z \rightarrow \infty} z \boldsymbol{e}\left(H_{1}^{-1}(z)\right)^{\top} \boldsymbol{u} \\
& =2 \lim _{t \rightarrow 1} H_{1}(t)\left(T_{0}(t), \ldots, T_{n-1}(t)\right) \check{\boldsymbol{u}}=-4 \mathrm{e}^{\mathrm{i} \pi / 6} \lim _{t \rightarrow 1} \frac{1}{t-1}\left(T_{0}(t), \ldots, T_{n-1}(t)\right) \check{\boldsymbol{u}} \\
& =-4 \mathrm{e}^{\mathrm{i} \pi / 6}\left(T_{0}^{\prime}(1), \ldots, T_{n-1}^{\prime}(1)\right) \check{\boldsymbol{u}}=-4 \mathrm{e}^{\mathrm{i} \pi / 6} \boldsymbol{e}_{n}^{\top} D \boldsymbol{u},
\end{aligned}
$$

where $D$ denotes the Chebyshev differentiation matrix (applicable in $\mathcal{O}(n \log n$ ) time using the DCT [6]) and we used the fact that $\boldsymbol{e}_{n}^{\top} \boldsymbol{u}$ is zero to employ L'Hopital's rule. In other words, we need only compute the derivative of the function mapped to the unit interval.

The right-hand side of Figure 2 demonstrates the convergence of $u_{n}$. Because we are differentiating, we lose approximately two digits of accuracy when moving from the solution of the Riemann-Hilbert problem to the solution of the differential equation. Thus, whereas the left-hand graph achieved roughly 13 digits of accuracy, the right hand graph only achieves roughly 11 digits of accuracy. In Figure 3 we plot solutions for two choices of $\left(s_{1}, s_{2}, s_{3}\right)$. See Figure 4 for the solution with $\left(s_{1}, s_{2}, s_{3}\right)=(1+\mathrm{i},-2,1-\mathrm{i})$.

\section{Initial Conditions}

So far, we have defined a unique solution to the homogeneous Painlevé II equation by specifying the constants $\left(s_{1}, s_{2}, s_{3}\right)$, in analogue to the analytic development in $[\mathbf{1 2}, \mathbf{1 0}]$. This is in contrast to what one would normally consider defining a unique solution to a differential 

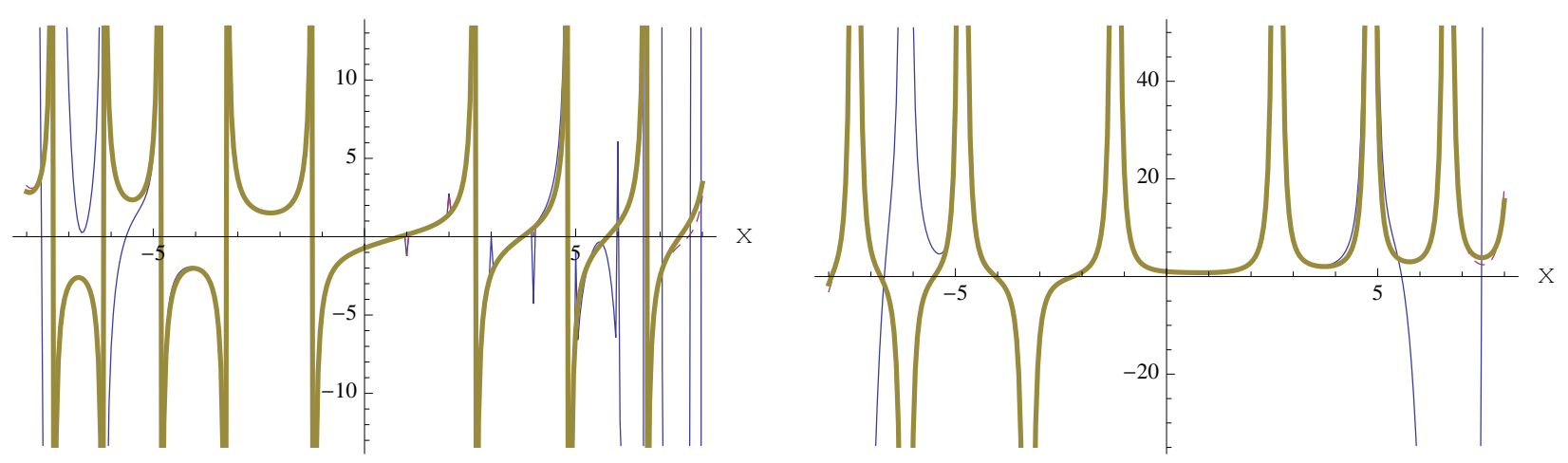

Figure 4: For $\left(s_{1}, s_{2}, s_{3}\right)=(1+\mathrm{i},-2,1-\mathrm{i})$, a plot of the real part of $u_{n}$ (left graph) and its derivative (right graph) for $n=50$ (plain), 100 (dashed) and 150 (thick).

equation: initial conditions, say, at $x=0$. Given the set $\left(s_{1}, s_{2}, s_{3}\right)$, we have already seen how we can use our approach to determine $u(x)$. But we can go one step further and determine $u^{\prime}(x)$ as well. Note that

$$
u^{\prime}(x)=2 \frac{\mathrm{d}}{\mathrm{d} x} \lim _{z \rightarrow \infty} z \Phi^{(12)}(x ; z)=2 \lim _{z \rightarrow \infty} z \Phi_{x}^{(12)}(x ; z) .
$$

Since $\Phi(x ; \infty)=I$ for all $x$, it follows that $\Phi_{x}(x ; \infty)=0$. Furthermore, differentiating $\Phi^{+}=\Phi^{-} G$ we obtain:

$$
\Phi_{x}^{+}-\Phi_{x}^{-} G=\Phi^{-} G_{x} \quad \text { and } \quad \Phi_{x}(\infty)=0 .
$$

Now we already know how to compute $\Phi^{-}=I+\mathcal{M}^{-} V$, hence the right-hand side is known. Furthermore, the left-hand side of the equation is exactly the left side of (1.3). Indeed, if we let $\Phi_{x}=\mathcal{M} \tilde{V}$, then we obtain again $\tilde{V}_{\kappa}^{(11)}=\tilde{V}_{\kappa-1}^{(12)}=\tilde{V}_{\kappa}^{(21)}=\tilde{V}_{\kappa-1}^{(22)}=0$ for $\kappa=2,4,6$. Thus we have the exact same linear systems as before, only with a different right-hand side. In the first linear system, the new right-hand side is:

$$
\begin{array}{r}
2 \mathrm{i} s_{1} \operatorname{diag}\left(\overline{\boldsymbol{z}}_{1}\right) \operatorname{diag}\left(\mathrm{e}^{8 \mathrm{i} / 3 \bar{z}_{1}^{3}+2 \mathrm{i} x \bar{z}_{1}}\right)\left[M_{6} \overline{\boldsymbol{v}}_{2}^{(12)}+M_{4} \overline{\boldsymbol{v}}_{4}^{(12)}+M_{2} \overline{\boldsymbol{v}}_{6}^{(12)}\right], \\
-2 \mathrm{i} s_{2} \operatorname{diag}\left(\overline{\boldsymbol{z}}_{2}\right) \operatorname{diag}\left(\mathrm{e}^{\left.-8 \mathrm{i} / 3 \overline{\boldsymbol{z}}_{2}^{3}-2 \mathrm{i} x \overline{\boldsymbol{z}}_{2}\right)}\left[M_{2} \overline{\boldsymbol{v}}_{1}^{(11)}+M_{6} \overline{\boldsymbol{v}}_{3}^{(11)}+M_{4} \overline{\boldsymbol{v}}_{5}^{(11)}\right],\right. \\
\vdots \\
-2 \mathrm{i} s_{2} \operatorname{diag}\left(\overline{\boldsymbol{z}}_{5}\right) \operatorname{diag}\left(\mathrm{e}^{\left.8 \mathrm{i} / 3 \overline{\boldsymbol{z}}_{5}^{3}+2 \mathrm{i} x \overline{\boldsymbol{z}}_{5}\right)}\left[M_{4} \overline{\boldsymbol{v}}_{2}^{(12)}+M_{2} \overline{\boldsymbol{v}}_{4}^{(12)}+M_{6} \overline{\boldsymbol{v}}_{6}^{(12)}\right],\right. \\
2 \mathrm{i} s_{1} \operatorname{diag}\left(\overline{\boldsymbol{z}}_{6}\right) \operatorname{diag}\left(\mathrm{e}^{-8 \mathrm{i} / 3 \overline{\boldsymbol{z}}_{6}^{3}-2 \mathrm{i} x \overline{\boldsymbol{z}}_{6}}\right)\left[M_{6} \overline{\boldsymbol{v}}_{1}^{(11)}+M_{4} \overline{\boldsymbol{v}}_{3}^{(11)}+M_{2} \overline{\boldsymbol{v}}_{5}^{(11)}\right] .
\end{array}
$$

In short, it is very inexpensive to compute $u^{\prime}(x)$ whenever $u(x)$ has already been computed using the Riemann-Hilbert formulation. This allows us to map $\left(s_{1}, s_{2}, s_{3}\right)$ to the equivalent initial conditions $u(x), u^{\prime}(x)$. 
We can use this approach to compare the approximation derived from the RiemannHilbert formulation to a standard ODE solver. We determine that the initial conditions for $\left(s_{1}, s_{2}, s_{3}\right)=(1+\mathrm{i},-2,1-\mathrm{i})$ are approximately (to about 10 digits accuracy)

$$
u(0) \approx-0.7233727039 \text { and } u^{\prime}(0) \approx 1.019298669 .
$$

Consider Figure 4, where we plot approximate solutions for this choice of constants. Note the presence of multiple poles. Unlike an ODE solver, which cannot possibly integrate past

a pole, our numerical Riemann-Hilbert approach is only affected by the pole when trying to evaluate close to the pole itself. For values of $x$ bounded away from the first pole (say, $x<2$ ), we can compare $u_{n}$ to MATHEMATICA's adaptive ODE solver NDSolve using the computed initial conditions and extra precision arithmetic. In particular, $u_{150}$ matches this computed solution to 10 digits, which is as most as can be expected given the limited accuracy of the initial conditions.

Remark: Of course, there is no guarantee of NDSolve's accuracy, as MATHEMATICA is a black box system, but we use it as it allows high precision arithmetic to hopefully achieve an approximation to machine precision accuracy. We leave it to the reader to decide whether this comparison validates the numerical Riemann-Hilbert approach by comparing it to NDSolve's approximation, or vice-versa.

In applications, one would typically want to find the direct transformation [8]: given initial conditions $u(0)$ and $u^{\prime}(0)$, determine $\left(s_{1}, s_{2}, s_{3}\right)$. But having a map from $\left(s_{1}, s_{2}, s_{3}\right)$ to the initial conditions $u(0)$ and $u^{\prime}(0)$ means that determining the inverse map can likely be found using optimization techniques. Indeed, we benefit from the fact that much of the work in constructing the linear system in Section 4 can be reused for different choices of $\left(s_{1}, s_{2}, s_{3}\right)$. We, however, leave this step as a future problem.

Remark: As far as I am aware, this is the only known approach of computing the initial conditions associated with the constants $\left(s_{1}, s_{2}, s_{3}\right)$. Since the constants $\left(s_{1}, s_{2}, s_{3}\right)$ determine the asymptotics of the solution, this would mean that this is the only way of connecting asymptotics with initial conditions.

\section{Condition number}

As $x$ increases, the jump function $G$ becomes increasingly oscillatory, hence it is sensible that the rate of convergence deteriorates. However, we also saw in numerical experiments that stability is lost. We now explain this behaviour by investigating the growth of the condition number of the linear system. In the left-hand side of Figure 5, we plot the growth of the condition number as $n$ increases for several choices of $x$, for $\left(s_{1}, s_{2}, s_{3}\right)=(1,2,1 / 3)$. 

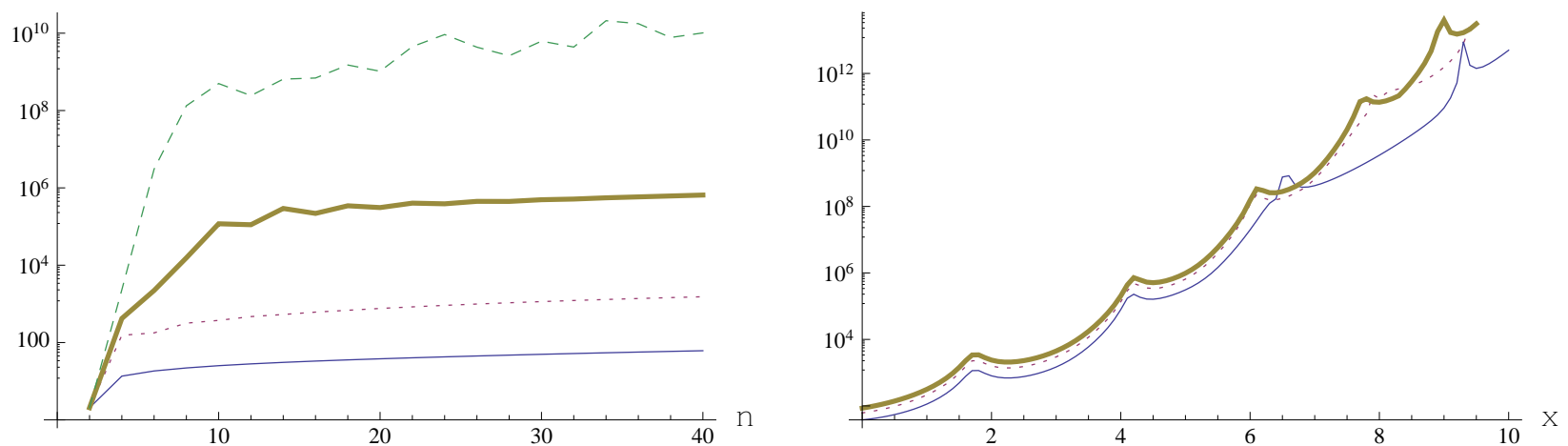

Figure 5: For $\left(s_{1}, s_{2}, s_{3}\right)=(1,2,1 / 3)$, the condition number of the first linear system, on the left for $x=0$ (plain), 2.5 (dotted), 5 (thick) and 7.5 (dashed) and on the right for $n=20$ (plain), 40 (dotted) and 60 (thick).

For each value of $x$, the condition number appears to grow linearly, which is quite good as the approximation converges spectrally. Unfortunately, as seen in the right-hand size of Figure 5, increasing $x$ causes exponential increase in the condition number! Thus the condition number quickly reaches the point where not even a single digit of accuracy can be achieved.

At first, this problem seems devastating to the approach: an exponentially increasing condition number makes the linear system unusable even for modest $n$, and to resolve the oscillations in the solution for large $x$ would require large $n$. However, consider for a moment the following contour representation for solutions to the Airy equation $[\mathbf{1 2}]$ :

$$
\left(s_{2} \int_{\Gamma_{2}}+s_{4} \int_{\Gamma_{4}}+s_{6} \int_{\Gamma_{6}}\right) \mathrm{e}^{-8 \mathrm{i} / 3 z^{3}-2 \mathrm{i} x z} \mathrm{~d} z \quad \text { for } \quad s_{2}+s_{4}+s_{6}=0 .
$$

The choice $\left(s_{2}, s_{4}, s_{6}\right)=\left(0,-\frac{1}{\pi}, \frac{1}{\pi}\right)$ is equivalent to the contour integral representation of $\operatorname{Ai}(x)[\mathbf{1 6}]$. This representation suffers from similar numerical issues as our linear system: $\mathrm{e}^{-8 \mathrm{i} / 3 z^{3}-2 \mathrm{i} x z}$ grows exponentially large for fixed $z$ in $\Gamma_{2}$ or $\Gamma_{4}$ as $x \rightarrow-\infty$ (though eventually the super-exponential decrease of $\mathrm{e}^{-8 \mathrm{i} / 3 z^{3}}$ wins out to make the integrals finite). But we know $\operatorname{Ai}(x)$ is bounded as $x \rightarrow-\infty$, hence the oscillations must cancel. In other words, roundoff error makes this integral representation useless. In the case of the Airy equation, we know how to resolve this issue: deform the contour through the saddle points at $\pm \frac{\mathrm{i} \sqrt{x}}{2}$ so that the contour avoids areas of the complex plane where the integrand exhibits exponential growth. This can be taken one step further so that the contour runs precisely along the path of steepest descent, thus not only avoiding exponential increase, but also oscillations all-together [11]. This has the added benefit that we do not need to increase the number of quadrature points as $|x| \rightarrow \infty$.

It is now clear how to resolve the conditioning problems for our linear system: deform the curve $\Gamma$ so it avoids the sectors of exponential growth by passing through the saddle points 

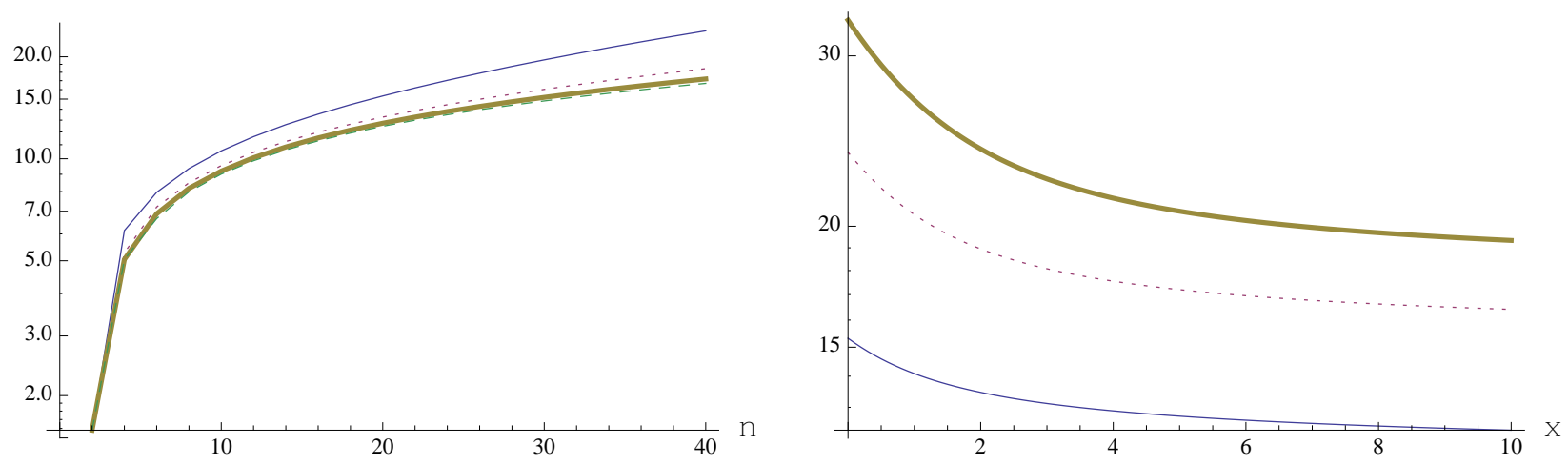

Figure 6: For $\left(s_{1}, s_{2}, s_{3}\right)=(1,0,-1)$, the condition number of the first linear system, on the left for $x=0$ (plain), 2.5 (dotted), 5 (thick) and 7.5 (dashed) and on the right for $n=20$ (plain), 40 (dotted) and 60 (thick).

of $G$. Moreover, in analogue to the integral case, we could even deform the contour along the path of steepest descent, thus avoiding oscillations. This path of steepest descent has already been computed for the derivation of the asymptotics of solutions to the homogeneous Painlevé II equation [8]. Now to apply our approach, if the steepest descent path is denoted $\Gamma^{\mathrm{SD}}$, then we would need to compute $\mathcal{M}_{\Gamma \mathrm{SD}}$. This could possibly be achieved by conformally mapping each of the pieces which make up $\Gamma^{\mathrm{SD}}$ to the unit interval. However, we do not necessarily need the exact steepest descent curve, as an approximate path will have only minor oscillations. Thus we could alternatively approximate $\Gamma^{\mathrm{SD}}$ by a linear interpolate. As we know how to compute $\mathcal{M}$ over line segments in the complex plane, we should be able to successfully apply our numerical Riemann-Hilbert approach. Again, we leave this as a future problem.

Without using the path of steepest descent, we can still demonstrate this phenomena by choosing $x$ and $\left(s_{1}, s_{2}, s_{3}\right)$ to avoid the exponential increase. When $x$ is real and positive, the only curve which see exponential increase as $x \rightarrow \infty$ are $\Gamma_{2}$ and $\Gamma_{5}$. Thus if $s_{2}=0$ then the exponential increase is avoided. As Figure 6 demonstrates, the condition number is now well behaved.

\section{Closing remarks}

We have demonstrated that a Riemann-Hilbert formulation is not just useful as an analytical tool, but also as a numerical one, by successfully computing solutions to the homogeneous Painlevé II equation. This could potentially lay the groundwork for the construction of a toolbox for computing Painlevé equations. Then Painlevé transcendentals would indeed be the true analogues of linear special functions such as the Airy equation: not only useful for analytical expressions, but efficient for practical computations as well.

In Figure 7 we depict the curves $\Gamma$ for the first five Painlevé Riemann-Hilbert problems, including the inhomogeneous Painlevé II equation. The important characteristic to note 

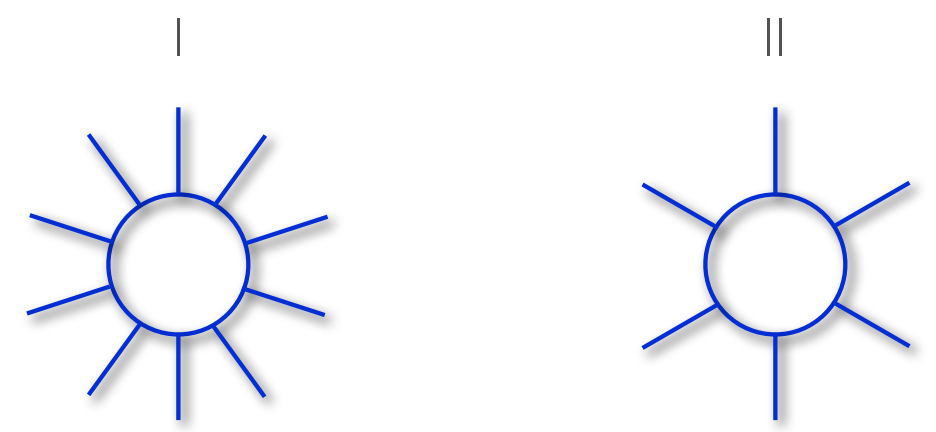

III
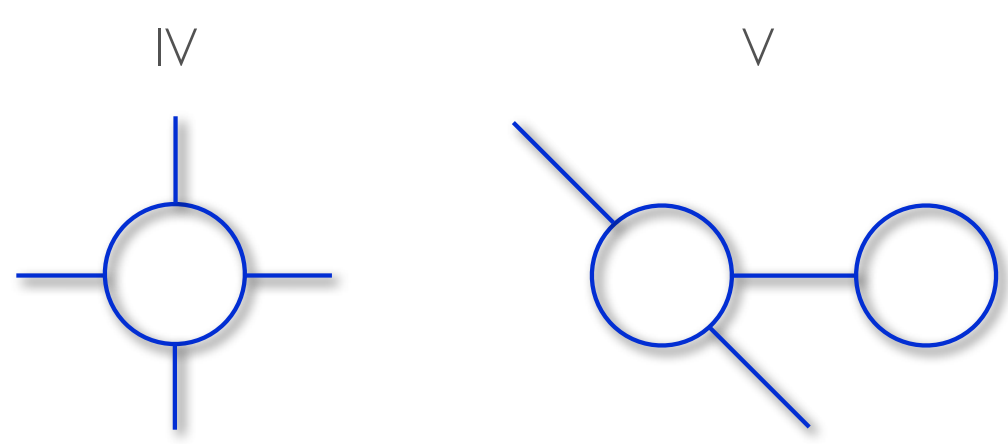

Figure 7: A depiction of the curves $\Gamma$ associated with the Painlevé $\mathrm{I}-\mathrm{V}$ equations.

is that they consist of a union of curves which each can be conformally mapped to the unit interval using Möbius transformations: rays, arcs and line segments. Thus the general approach of Algorithm 1.4 can already be implemented for these equations.

Because of the numerical problems described in Section 7, this approach is currently not practical for large $x$, though it is likely that using the path of steepest descent will rectify this issue. However, it is practical for small $x$, in particular for computing initial conditions. Thus it can already be used to connect asymptotic formulæ - which are known in terms of the constants $\left(s_{1}, s_{2}, s_{3}\right)$ - to initial conditions. Thus a simple, practical numerical implementation for the computation of a solution to the homogeneous Painlevé II transcendent for large and small $|x|$ is now straightforward: use the proposed approach for small $x$, use asymptotic formulæ for large $|x|$ and use an ODE solver to extend these two regimes to moderate $x$.

Acknowledgments: I would like to thank Peter Clarkson, Toby Driscoll, Thanasis Fokas, Nick Trefethen, Andy Wathen and Andre Weideman for their valuable advice. 


\section{References}

[1] Ablowitz, M.J. and Segur, H., Solitons and the inverse scattering transform, Society for Industrial Mathematics, 2006.

[2] Abramowitz, M. and Stegun, I., Handbook of Mathematical Functions, National Bureau of Standards Appl. Math. Series, \#55, U.S. Govt. Printing Office, Washington, D.C., 1970.

[3] Aksenov, S., Savageau, M.A., Jentschura, U.D., Becher, J., Soff, G. and Mohr, P.J., Application of the combined nonlinear-condensation transformation to problems in statistical analysis and theoretical physics, Comp. Phys. Comm. 150 (2003), 1-20.

[4] Bateman, H., Higher Transcendental Functions, McGraw-Hill, New York, 1953.

[5] Berrut, J.-P. and Trefethen, L.N., Barycentric lagrange interpolation, SIAM Review 46 (2004), 501-517.

[6] Boyd, J.P., Chebyshev and Fourier spectral methods, Dover Pubns, 2001.

[7] Deift, P., Orthogonal polynomials and random matrices: a Riemann-Hilbert approach, American Mathematical Society, 2000.

[8] Deift, P. and Zhou, X., Asymptotics for the Painlevé II equation, Communications on Pure and Applied Mathematics 48 (1995), 277.

[9] Deift, P. and Zhou, X., A steepest descent method for oscillatory Riemann-Hilbert problems, AMS 26 (1992), 119-124.

[10] Fokas, A.S., Its, A.R., Kapaev, A.A. and Novokshenov,V.Y., Painlevé transcendents: the Riemann-Hilbert approach, American Mathematical Society, 2006.

[11] Gil, A., Segura, J. and Temme, N.M., Numerical Methods for Special Functions, SIAM, 2007.

[12] Its, A.R., The Riemann-Hilbert problem and integrable systems, Notices AMS 50 (2003), 1389-1400.

[13] King, F.W., Hilbert Transforms: Volume 1, Cambridge University Press, 2009.

[14] Muskhelishvili, N.I., Singular integral equations, Groningen: Noordhoff (based on the second Russian edition published in 1946), 1953.

[15] Nasser, M.M.S., Numerical solution of the Riemann-Hilbert problem, Punjab University Journal of Mathematics 40 (2008), 9-29.

[16] Olver, F.W.J., Asymptotics and Special Functions, Academic Press, New York, 1974. 
[17] Olver, S., Computing the Hilbert transform and its inverse, preprint, NA-09/07, Maths Institute, Oxford University.

[18] Wegert, E., An iterative method for solving nonlinear Riemann-Hilbert problems, Journal of Computational and Applied Mathematics 29 (1990), 327.

[19] Wegmann, R., Discrete Riemann-Hilbert problems, interpolation of simply closed curves, and numerical conformal mapping, J. Comp. Appl. Maths 23 (1988), 323-352.

[20] Wegmann, R., An iterative method for the conformal mapping of doubly connected regions, Journal of computational and applied mathematics 14 (1986), 79-98. 